\title{
FICTITIOUS DOMAIN METHODS USING CUT ELEMENTS: III. A STABILIZED NITSCHE METHOD FOR STOKES' PROBLEM
}

\author{
ERIK BURMAN ${ }^{1}$ AND PETER HANSBO ${ }^{2}$
}

\begin{abstract}
We extend our results on fictitious domain methods for Poisson's problem to the case of incompressible elasticity, or Stokes' problem. The mesh is not fitted to the domain boundary. Instead boundary conditions are imposed using a stabilized Nitsche type approach. Control of the non-physical degrees of freedom, i.e., those outside the physical domain, is obtained thanks to a ghost penalty term for both velocities and pressures. Both inf-sup stable and stabilized velocity pressure pairs are considered.
\end{abstract}

Mathematics Subject Classification. 65N12, 65N30.

Received August 14, 2012. Revised August 8, 2013.

Published online April 22, 2014.

\section{INTRODUCTION}

In our previous work [14] we considered a fictitious domain method for Poisson's problem that used Nitsche's method to impose boundary conditions. Herein we will extend this approach to the case of Stokes' problem. The complication, compared with [14], is that the mutual satisfaction of the boundary conditions on the unfitted boundary and the discrete inf-sup condition leads to difficulties. This is not surprising since the discrete inf-sup condition requires a careful balancing of the spaces for velocity and pressure approximation and since the boundary can cut the elements in an almost arbitrary fashion, uniformity can not, in general, be guaranteed. In the present paper we follow the ideas of $[7,15]$. We assume either that the velocity-pressure pair used satisfies the discrete inf-sup condition in the interior of the domain or that a symmetric stabilization method is used. Stability up to the mesh boundary is then obtained by a ghost penalty term.

Since we obtain stability uniformly over the domain, we can prove that the condition number is bounded irrespectively of how the boundary cuts the mesh. Optimal convergence estimates are then obtained for smooth solutions using suitable extensions of the exact solution. Here it is important that the ghost-penalty terms are weakly consistent to the right order, but still ensures sufficient control of the non-physical degrees of freedom.

Previous work on fictitious domain methods for Stokes include the multiplier method of Girault, Glowinskin, and Pan [20] and the penalty method of Angot [3]. We remark that these methods employ continuous approximations which is a fundamental problem since there is a need to accommodate kinks in the derivative across the interface for optimal convergence. An exception is the fat boundary method by Maury and co-workers,

\footnotetext{
Keywords and phrases. Finite element methods, stabilized methods, penalty methods, Stokes' problem, fictitious domain.

1 Department of Mathematics, University College London, UK-WC1E 6BT United Kingdom

2 Department of Mechanical Engineering, Jönköping University, 55111 Jönköping, Sweden. peter.hansbo@jth.hj.se
} 
e.g., [10], in which a fixed point iteration scheme is employed to couple two different but dependent problem outside and inside the artificial boundary. We also refer to related work by Haslinger and Renard [22] and Amdouni, Moakher and Renard [2] using different stabilizations of Lagrange multiplier methods. Work on cut elements in mixed methods for Stokes (or incompressible elasticity), outside the realms of fictitious domains, include [7], where a stabilized P1P0 method was used, the X-FEM methods of Amdouni et al. [1], where the inf-sup stable P2P0 element was used, and of Legrain, Moës, and Huerta [23], who used the inf-sup stable MINI element.

\section{THE STOKES PROBLEM}

Let $\Omega$ be a bounded domain in $\mathbb{R}^{2}$ with polygonal boundary $\Gamma$ (possibly with smooth curved faces). The Stokes' problem takes the form, find the velocities $\boldsymbol{u}$ and the pressure $p$ such that

$$
\begin{aligned}
-\mu \Delta \boldsymbol{u}+\boldsymbol{\nabla} p & =\boldsymbol{f} & & \text { in } \Omega \\
\nabla \cdot \boldsymbol{u} & =0 & & \text { in } \Omega \\
\boldsymbol{u} & =0 & & \text { on } \Gamma .
\end{aligned}
$$

Here $\boldsymbol{f} \in\left[L^{2}(\Omega)\right]^{2}, \boldsymbol{\Delta} \boldsymbol{u}=\left[\Delta u_{1}, \ldots, \Delta u_{d}\right]$ Let $(u, v)_{X}$ denote the $L^{2}$-scalar product on $X$,

$$
(u, v)_{X}:=\int_{X} u v \mathrm{~d} x
$$

with associated norm $\|u\|_{0, X}:=(u, u)_{X}^{\frac{1}{2}}$. The Sobolev norms associated with the spaces $H^{k}(X)$ will be denoted by $\|u\|_{k, X}$. We let $L_{0}^{2}(\Omega)$ denote the functions in $L^{2}(\Omega)$ with zero average. Formally we obtain the weak formulation by multiplying (2.1) by a function $(\boldsymbol{v}, q) \in\left[H_{0}^{1}(\Omega)\right]^{2} \times L_{0}^{2}(\Omega)=V \times Q$ and integrating by parts, leading to the problem of finding $(\boldsymbol{u}, p) \in V \times Q$ such that

$$
(\mu \boldsymbol{\nabla} \boldsymbol{u}, \boldsymbol{\nabla} \boldsymbol{v})_{\Omega}-(p, \boldsymbol{\nabla} \cdot \boldsymbol{v})_{\Omega}+(q, \boldsymbol{\nabla} \cdot \boldsymbol{u})_{\Omega}=(\boldsymbol{f}, \boldsymbol{v})_{\Omega}, \text { for all }(\boldsymbol{v}, q) \in V \times Q,
$$

where

$$
(\nabla \boldsymbol{u}, \boldsymbol{\nabla v})_{\Omega}:=\int_{\Omega} \boldsymbol{\nabla u}: \nabla \boldsymbol{v} \mathrm{d} x .
$$

On more compact form the problems reads: find $(\boldsymbol{u}, p) \in V \times Q$ such that

$$
B[(\boldsymbol{u}, p),(\boldsymbol{v}, q)]=(\boldsymbol{f}, \boldsymbol{v})_{\Omega}, \text { for all }(\boldsymbol{v}, q) \in V \times Q,
$$

where

$$
B[(\boldsymbol{u}, p),(\boldsymbol{v}, q)]:=(\mu \boldsymbol{\nabla} \boldsymbol{u}, \boldsymbol{\nabla} \boldsymbol{v})_{\Omega}-(p, \boldsymbol{\nabla} \cdot \boldsymbol{v})_{\Omega}+(q, \boldsymbol{\nabla} \cdot \boldsymbol{u})_{\Omega} .
$$

For the purposes of the analysis we will assume that $\boldsymbol{u} \in\left[H^{\alpha}(\Omega)\right]^{2}, p \in H^{\beta}(\Omega)$, with $\alpha \geq 2$ and $\beta \geq 1$.

\section{The Finite ELEMENT FORMUlation}

In a standard finite element method the mesh is fitted to the boundary or interpolates the boundary to some suitable order. Instead we propose to solve (2.1) approximately on a family of quasi-uniform, conforming triangulations $\mathcal{T}_{h}$, such that $\bar{\Omega} \subset \mathcal{T}_{h}$ but $\mathcal{T}_{h} \not \subset \bar{\Omega}$. However for all triangles $K \in \mathcal{T}_{h}$ there holds $K \cap \Omega \neq \emptyset$ and we define the domain covered by $\mathcal{T}_{h}$ by $\Omega_{\mathcal{T}}:=\cup_{K \in \mathcal{T}_{h}} K$. In the analysis we use the notation $a \lesssim b$ for $a \leq C b$, where $C$ denotes a generic constant that may change at each occurrence, but is independent of $h$ and of the interface position under the assumptions made precise in following section.

We will use the following notation for mesh related quantities. Let $h_{K}$ be the diameter of $K$ and $h=$ $\max _{K \in \mathcal{T}_{h}} h_{K}$. By

$$
G_{h}:=\left\{K \in \mathcal{T}_{h}: K \cap \Gamma \neq \emptyset\right\}
$$


we denote the set of elements that are intersected by the boundary. For an element $K \in G_{h}$, let $\Gamma_{K}:=\Gamma \cap K$ be the part of $\Gamma$ intersecting $K$.

We make the following assumptions regarding the mesh and the boundary (from [21]).

- A1: We assume that the triangulation is non-degenerate, i.e. there exists $C>0$ such that,

$$
h_{K} / \rho_{K} \leq C \quad \forall K \in \mathcal{T}_{h}
$$

where $h_{K}$ is the diameter of $K$ and $\rho_{K}$ is the diameter of the largest ball contained in $K$.

- A2: We assume that $\Gamma$ intersects the boundary $\partial K$ of an element $K$ in $G_{h}$ exactly twice and each (open) edge at most once.

- A3: Let $\Gamma_{K, h}$ be the straight line segment connecting the points of intersection between $\Gamma$ and $\partial K$. We assume that $\Gamma_{K}$ is a function of length on $\Gamma_{K, h}$; in local coordinates

$$
\Gamma_{K, h}=\left\{(\xi, \eta): 0<\xi<\left|\Gamma_{K, h}\right|, \eta=0\right\}
$$

and

$$
\Gamma_{K}=\left\{(\xi, \eta): 0<\xi<\left|\Gamma_{K, h}\right|, \eta=\delta(\xi)\right\} .
$$

Since the curvature of $\Gamma$ is bounded almost everywhere, the assumptions A2 and A3 are always fulfilled on sufficiently fine meshes. These assumptions essentially demand that the interface is well resolved by the mesh.

We denote the set of interior faces of the triangles in $\mathcal{T}_{h}$ by $\mathcal{F}_{h}$. The faces of triangles in $G_{h}$, that are not on the mesh boundary $\partial \Omega_{\mathcal{T}}$ will be denoted $\mathcal{F}_{G}$,

$$
\mathcal{F}_{G}:=\left\{F \in \partial K \text { for some } K \in G_{h} \text { and } F \cap \partial \Omega_{\mathcal{T}} \neq F\right\} .
$$

Associated with $\mathcal{T}_{h}$ we have the finite element spaces

$$
\begin{gathered}
V_{h}=\left\{\boldsymbol{v} \in\left[C^{0}(\Omega)\right]^{2}:\left.\boldsymbol{v}\right|_{K} \in\left[P_{k}(K)\right]^{2}, \forall K \in \mathcal{T}_{h}\right\}, \\
Q_{h}=\left\{q \in L^{2}(\Omega): \int_{\Omega \backslash G_{h}} q \mathrm{~d} x=0 ;\left.q\right|_{K} \in P_{m}(K), \forall K \in \mathcal{T}_{h}\right\} .
\end{gathered}
$$

Remark 3.1. Herein we restrict the discussion to this simple choice of spaces; however, the extension of the results to other spaces, using for instance bubble enriched velocities, is immediate. The orders $m$ and $k$ above can be thought of as the highest full polynomial order of the space.

The finite element discretisation now takes the form: find $\left(\boldsymbol{u}_{h}, p_{h}\right) \in V^{h} \times Q^{h}$ such that

$$
B_{h}\left[\left(\boldsymbol{u}_{h}, p_{h}\right),\left(\boldsymbol{v}_{h}, q_{h}\right)\right]+S_{h}\left[\left(\boldsymbol{u}_{h}, p_{h}\right),\left(\boldsymbol{v}_{h}, q_{h}\right)\right]=\left(\boldsymbol{f}, \boldsymbol{v}_{h}\right),
$$

for all $\left(\boldsymbol{v}_{h}, q_{h}\right) \in V_{h} \times Q_{h}$, where

$$
B_{h}\left[\left(\boldsymbol{u}_{h}, p_{h}\right),\left(\boldsymbol{v}_{h}, q_{h}\right)\right]:=a_{h}\left(\boldsymbol{u}_{h}, \boldsymbol{v}_{h}\right)+b_{h}\left(p_{h}, \boldsymbol{v}_{h}\right)-b_{h}\left(q_{h}, \boldsymbol{u}_{h}\right)
$$

where

$$
\begin{aligned}
a_{h}\left(\boldsymbol{u}_{h}, \boldsymbol{v}_{h}\right)= & \int_{\Omega} \mu \boldsymbol{\nabla} \boldsymbol{u}_{h}: \boldsymbol{\nabla} \boldsymbol{v}_{h} \mathrm{~d} x-\int_{\Gamma}\left(\mu \boldsymbol{\nabla} \boldsymbol{u}_{h} \boldsymbol{n}\right) \cdot \boldsymbol{v}_{h} \mathrm{~d} s \\
& -\int_{\Gamma}\left(\mu \boldsymbol{\nabla} \boldsymbol{v}_{h} \boldsymbol{n}\right) \cdot \boldsymbol{u}_{h} \mathrm{~d} s+\sum_{K \in G_{h}} \int_{\Gamma_{K}} \gamma_{\mu} \mu h_{K}^{-1} \boldsymbol{u}_{h} \cdot \boldsymbol{v}_{h} \mathrm{~d} s
\end{aligned}
$$

and

$$
b_{h}\left(p_{h}, \boldsymbol{v}_{h}\right)=-\int_{\Omega} p_{h} \boldsymbol{\nabla} \cdot \boldsymbol{v}_{h} \mathrm{~d} x+\int_{\Gamma} p_{h} \boldsymbol{v}_{h} \cdot \boldsymbol{n} \mathrm{d} s .
$$


Here, $\gamma_{\mu} \in \mathbb{R}^{+}$is a term designed to ensure coercivity of the bilinear form $a_{h}(\cdot, \cdot), c f$. Lemma 4.2. The term

$$
S_{h}\left[\left(\boldsymbol{u}_{h}, p_{h}\right),\left(\boldsymbol{v}_{h}, q_{h}\right)\right]:=\gamma_{p} s_{h}\left(p_{h}, q_{h}\right)+\gamma_{g} g_{h}\left[\left(\boldsymbol{u}_{h}, p_{h}\right),\left(\boldsymbol{v}_{h}, q_{h}\right)\right],
$$

where $\gamma_{p} \geq 0$ and $\gamma_{g} \in \mathbb{R}^{+}$are free parameters, denotes a stabilization term consisting of one part $s\left(p_{h}, q_{h}\right)$ that ensures the inf-sup condition in the bulk in case it is not satisfied by the spaces $V_{h} \times Q_{h}$, and another part $g_{h}\left[\left(\boldsymbol{u}_{h}, p_{h}\right),\left(\boldsymbol{v}_{h}, q_{h}\right)\right]:=\mu g_{u}\left(\boldsymbol{u}_{h}, \boldsymbol{v}_{h}\right)+\mu^{-1} g_{p}\left(p_{h}, q_{h}\right)$ that denotes a ghost penalty term active in the interface zone only. The subscripts $u$ and $p$ denote that different weights are used for velocities and pressures in the penalty term. The stabilization operator will be assumed to satisfy the following upper bounds,

$$
S_{h}\left[\left(\boldsymbol{v}_{h}, q_{h}\right),\left(\boldsymbol{v}_{h}, 0\right)\right] \lesssim \mu\left\|\nabla \boldsymbol{v}_{h}\right\|_{0, \Omega_{\mathcal{T}}}^{2}
$$

and

$$
S_{h}\left[\left(\boldsymbol{v}_{h}, q_{h}\right),\left(0, q_{h}\right)\right] \lesssim \mu^{-1}\left\|q_{h}\right\|_{0, \Omega_{\mathcal{T}}}^{2} .
$$

The role of the ghost penalty term is to enforce the following strengthened stability

$$
\begin{aligned}
\mu\left\|\boldsymbol{\nabla} \boldsymbol{v}_{h}\right\|_{0, \Omega_{\mathcal{T}}}^{2} & \lesssim \mu\left\|\boldsymbol{\nabla} \boldsymbol{v}_{h}\right\|_{0, \Omega}^{2}+g_{h}\left[\left(\boldsymbol{v}_{h}, 0\right),\left(\boldsymbol{v}_{h}, 0\right)\right] \\
& \text { and } \\
\mu^{-1}\left\|q_{h}\right\|_{0, \Omega_{\mathcal{T}}}^{2} & \lesssim \mu^{-1}\left\|q_{h}\right\|_{0, \Omega \backslash G_{h}}^{2}+g_{h}\left[\left(0, q_{h}\right),\left(0, q_{h}\right)\right]
\end{aligned}
$$

for $\left(\boldsymbol{v}_{h}, q_{h}\right) \in V_{h} \times Q_{h}$. The operator $g_{h}[(\cdot, \cdot),(\cdot, \cdot)]$ must satisfy certain weak consistency properties and continuity properties that will be specified in the following. Examples of valid ghost penalty terms have been given in $[6,14,16]$ and will be recalled in the next section.

We assume that the following stability condition holds for $V_{h} \times Q_{h}$, for all $p_{h} \in Q_{h}$ there exists $\boldsymbol{v}_{p h} \in$ $V_{h} \cap H_{0}^{1}\left(\Omega \backslash G_{h}\right)$ such that

and

$$
\left\|\boldsymbol{v}_{p h}\right\|_{1, \Omega \backslash G_{h}} \leq c_{p 1} \mu^{-1}\left\|p_{h}\right\|_{0, \Omega \backslash G_{h}}
$$

$$
c_{p 2} \mu^{-1}\left\|p_{h}\right\|_{0, \Omega \backslash G_{h}}^{2} \leq b_{h}\left(p_{h}, \boldsymbol{v}_{p h}\right)+s\left(p_{h}, p_{h}\right),
$$

where $s\left(p_{h}, p_{h}\right)$ is a stabilization operator that may be zero if the velocity-pressure spaces are chosen so as to satisfy the relations (3.5) and (3.6) without it. Conditions (3.5) and (3.6) simply mean that we either use an inf-sup stable velocity pressure pair in the interior of the domain, or a pressure stabilized finite element method with symmetric stabilization. For relevant results on inf-sup stable elements we refer for instance to $[9,11,13]$ and for stabilized finite element methods that may be used in the present context to $[5,8,12,15,16,19]$.

Remark 3.2. Note that the displacements and the pressures are defined also in the domain $\mathcal{T}_{h} \backslash \Omega$ where they have no physical significance. Only the penalty term is active in this zone.

Remark 3.3. Since the pressure is determined only up to a constant we have imposed the condition

$$
\int_{\Omega \backslash G_{h}} q_{h} \mathrm{~d} x=0, \quad \forall q_{h} \in Q_{h} .
$$

This is convenient both from the point of view of analysis and of implementation,

The formulation (3.1) satisfies the following consistency relation.

Lemma 3.4 (Galerkin orthogonality). Let $\left(\boldsymbol{u}_{h}, p_{h}\right)$ be the solution of the finite element formulation (3.1) and $(\boldsymbol{u}, p) \in\left[H^{2}(\Omega)\right]^{2} \times H^{1}(\Omega)$ be the solution of $(2.3)$. Then

$$
B_{h}\left[\left(\boldsymbol{u}_{h}-\boldsymbol{u}, p_{h}-p\right),\left(\boldsymbol{v}_{h}, q_{h}\right)\right]=-S_{h}\left[\left(\boldsymbol{u}_{h}, p_{h}\right),\left(\boldsymbol{v}_{h}, q_{h}\right)\right] \quad \forall\left(\boldsymbol{v}_{h}, q_{h}\right) \in V_{h} \times Q_{h} .
$$

Proof. Using the formulation (3.1) we may write

$$
a_{h}\left(\boldsymbol{u}, \boldsymbol{v}_{h}\right)+b_{h}\left(p, \boldsymbol{v}_{h}\right)-b_{h}\left(q_{h}, \boldsymbol{u}\right)=\left(\boldsymbol{f}, \boldsymbol{v}_{h}\right) .
$$

The claim follows by noting that $b_{h}\left(q_{h}, \boldsymbol{u}\right)=0$ and by integrating by parts in the remaining two terms on the left hand side and using equation (2.1). 


\subsection{Examples of ghost penalty operators}

Depending on what polynomial order is used in the approximation spaces the ghost penalty operator must be designed differently. For high order polynomial approximation the ghost penalty term must give control of all polynomial orders. This can be achieved by adding a penalty on the jump of derivatives of all orders,

$$
g_{\sigma}\left(v_{h}, w_{h}\right):=\sum_{F \in \mathcal{F}_{G}} \sum_{j=1}^{k} h_{K}^{2(j-1)+\sigma} \int_{F}\left[D^{j} v_{h}\right]\left[D^{j} w_{h}\right] \mathrm{d} s .
$$

Here $[v]$ denotes $\lim _{\varepsilon \rightarrow 0^{+}}\left(v\left(\boldsymbol{x}+\varepsilon \boldsymbol{n}_{F}\right)-v\left(\boldsymbol{x}-\varepsilon \boldsymbol{n}_{F}\right)\right)$, with $\boldsymbol{n}_{F}$ normal to $F$, and $D^{j} v$ denotes the partial derivative of order $j$ in the normal direction. The evaluation of high order derivatives can be avoided since this operator is equivalent to a local projection stabilization operator: for each face $F \in \mathcal{F}_{G}$ we introduce the pair of elements $K$ and $K^{\prime}$ such that $F=K \cap K^{\prime}$ and set $\hat{K}_{F}:=K \cup K^{\prime}$. The penalty operator is then defined over all $\hat{K}_{F}$ as follows

$$
g_{\sigma}\left(v_{h}, w_{h}\right):=\sum_{F \in \mathcal{F}_{G}}\left(h^{\sigma-3}\left(v_{h}-G_{F} v_{h}\right), w_{h}\right)_{\hat{K}_{F}},
$$

where $G_{F} u_{h}$ is a projection onto a polynomial on the two elements having $F$ as a face defined by $G_{F} v_{h} \in W_{l}\left(\hat{K}_{F}\right)$ such that

$$
\left(\tau^{-1} G_{F} v_{h}, z_{h}\right)_{\hat{K}_{F}}=\left(\tau^{-1} v_{h}, z_{h}\right)_{\hat{K}_{F}}, \quad \forall z_{h} \in W_{l}\left(\hat{K}_{F}\right) .
$$

where the projection space $W_{l}\left(\hat{K}_{F}\right)$ is defined by

$$
W_{l}\left(\hat{K}_{F}\right):=\left\{z_{h} \in P_{l}\left(\hat{K}_{F}\right)\right\}, l \geq 0 .
$$

Observe that $l$ must be chosen larger than or equal to $k$ for the velocities and larger than or equal to $m-1$ for the pressure in order to ensure weak consistency.

The simplest case is obtained for $H^{1}$-conforming piecewise affine approximation, where the form (3.9) reduces to a penalty on the jump of the normal gradient between adjacent elements. The jump operator is defined by

$$
g_{\sigma}\left(v_{h}, w_{h}\right):=\sum_{F \in \mathcal{F}_{G}} \int_{F} h^{\sigma}\left[\nabla v_{h} \boldsymbol{n}_{F}\right] \cdot\left[\boldsymbol{\nabla} w_{h} \boldsymbol{n}_{F}\right] \mathrm{d} s
$$

with the natural, component wise extension to vector valued functions $\boldsymbol{v}:=\left(v_{1 h}, v_{2 h}\right)$,

$$
g_{\sigma}\left(\boldsymbol{v}_{h}, \boldsymbol{w}_{h}\right):=\sum_{i=1}^{2} \sum_{F \in \mathcal{F}_{G}} \int_{F} h^{\sigma}\left[\boldsymbol{\nabla} v_{i h} \boldsymbol{n}_{F}\right] \cdot\left[\boldsymbol{\nabla} w_{i h} \boldsymbol{n}_{F}\right] \mathrm{d} s .
$$

Then the ghost penalty operator takes the form

$$
g_{h}\left[\left(\boldsymbol{u}_{h}, p_{h}\right),\left(\boldsymbol{w}_{h}, q_{h}\right)\right]:=\mu g_{1}\left(\boldsymbol{u}_{h}, \boldsymbol{w}_{h}\right)+\mu^{-1} g_{3}\left(p_{h}, q_{h}\right) .
$$

Note the different scaling in $h$ for the operators acting on the velocity and the pressure. This is because the pressure is controlled in the $L^{2}$-norm and the velocity in the $H^{1}$-norm. It is easy to prove that the bounds $(3.2)$ and (3.3) hold.

For further details on these approaches we refer to $[6,17]$. Numerical examples, both using gradient penalty and the local projection approach, will be given in Section 7 . 


\subsection{Examples of pressure stabilization operators}

Most symmetric pressure stabilization methods known in the literature may be used. For piecewise affine approximations it may be particularly appealing to apply the interior penalty stabilization of [15] where

$$
s_{h}\left(p_{h}, q_{h}\right):=\mu^{-1} \sum_{F \in \mathcal{F}_{h}} \int_{F} h_{F}^{3}\left[\boldsymbol{\nabla} p_{h} \cdot \boldsymbol{n}_{F}\right] \cdot\left[\boldsymbol{\nabla} q_{h} \cdot \boldsymbol{n}_{F}\right] \mathrm{d} s
$$

since in that case all stabilization of the pressure is handled in a unified fashion. Otherwise the classical Brezzi-Pitkäranta stabilization [12] will work, as well as local projection type stabilizations [5], or other pressure projection stabilizations $[15,16,19]$. The Brezzi-Pitkäranta stabilization may also serve as ghost penalty term for the pressure, but has too poor consistency properties to be used for the velocities. Also in this case it is easy to prove the upper bound (3.3).

\section{Norms, CONTINUITY AND STABILITY}

We will use the following norms on the trace of a function on $\Gamma$.

$$
\begin{aligned}
\|\boldsymbol{v}\|_{1 / 2, h, \Gamma}^{2} & :=\sum_{K \in G_{h}} h_{K}^{-1}\|\boldsymbol{v}\|_{0, \Gamma_{K}}^{2}, \\
\|\boldsymbol{v}\|_{-1 / 2, h, \Gamma}^{2} & :=\sum_{K \in G_{h}} h_{K}^{1}\|\boldsymbol{v}\|_{0, \Gamma_{K}}^{2} .
\end{aligned}
$$

We note for future reference that

$$
(\boldsymbol{u}, \boldsymbol{v})_{\Gamma} \leq\|\boldsymbol{u}\|_{1 / 2, h, \Gamma}\|\boldsymbol{v}\|_{-1 / 2, h, \Gamma} .
$$

We also define norms associated with the discrete velocity and pressure approximations respectively:

$$
\left\|\boldsymbol{u}_{h}\right\|_{V, \mathcal{T}}^{2}:=\left\|\mu^{\frac{1}{2}} \nabla \boldsymbol{u}_{h}\right\|_{0, \Omega_{\mathcal{T}}}^{2}+\left\|\mu^{\frac{1}{2}} \boldsymbol{u}_{h}\right\|_{1 / 2, h, \Gamma}^{2}
$$

and

$$
\left\|p_{h}\right\|_{Q, \mathcal{T}}:=\left\|\mu^{-\frac{1}{2}} p_{h}\right\|_{0, \Omega_{\mathcal{T}}} .
$$

For the analysis we will use the following mesh dependent norms defined for functions in $\left[H^{2}(X)\right]^{2}+V_{h}$ or $\left[H^{2}(X)\right]^{2} \times H^{1}(X)$

$$
\|\boldsymbol{v}\|_{X}^{2}:=\left\|\mu^{\frac{1}{2}} \boldsymbol{\nabla} \boldsymbol{v}\right\|_{0, X}^{2}+\left\|\mu^{\frac{1}{2}} \nabla \boldsymbol{v} \boldsymbol{n}\right\|_{-1 / 2, h, \Gamma}^{2}+\left\|\mu^{\frac{1}{2}} \boldsymbol{v}\right\|_{1 / 2, h, \Gamma}^{2}
$$

and

$$
\|(\boldsymbol{u}, p)\|_{*}^{2}:=\|\boldsymbol{u}\|_{\Omega}^{2}+\left\|\mu^{-\frac{1}{2}} p\right\|_{0, \Omega}^{2}+\left\|\mu^{-\frac{1}{2}} p\right\|_{-1 / 2, h, \Gamma}^{2}
$$

and on $V_{h} \times Q_{h}$ the norm

$$
\left\|\left(\boldsymbol{u}_{h}, p_{h}\right)\right\|_{h}^{2}:=\left\|\boldsymbol{u}_{h}\right\|_{V, \mathcal{T}}^{2}+\left\|p_{h}\right\|_{Q, \mathcal{T}}^{2}+S_{h}\left[\left(\boldsymbol{u}_{h}, p_{h}\right),\left(\boldsymbol{u}_{h}, p_{h}\right)\right] .
$$

Lemma 4.1 (Continuity of $B_{h}[(\cdot, \cdot),(\cdot, \cdot)]$ and $S_{h}[(\cdot, \cdot),(\cdot, \cdot))$. Let $\boldsymbol{v}_{h}, \boldsymbol{w}_{h} \in V_{h}, \boldsymbol{u} \in V+V_{h}, q_{h}, y_{h} \in Q_{h}$ and $p \in Q+Q_{h}$. Then there holds,

$$
\begin{gathered}
B_{h}\left[(\boldsymbol{u}, p),\left(\boldsymbol{v}_{h}, q_{h}\right)\right] \lesssim\|\|(\boldsymbol{u}, p)\|\|_{*}\left\|\left(\boldsymbol{v}_{h}, q_{h}\right)\right\|_{h}, \\
S_{h}\left[\left(\boldsymbol{v}_{h}, q_{h}\right),\left(\boldsymbol{w}_{h}, y_{h}\right)\right] \leq S_{h}\left[\left(\boldsymbol{v}_{h}, q_{h}\right),\left(\boldsymbol{v}_{h}, q_{h}\right)\right]^{1 / 2} S_{h}\left[\left(\boldsymbol{w}_{h}, y_{h}\right),\left(\boldsymbol{w}_{h}, y_{h}\right)\right]^{1 / 2} .
\end{gathered}
$$


Proof. It follows from the Cauchy-Schwarz inequality and the trace inequality that

$$
\begin{aligned}
a_{h}\left(\boldsymbol{u}, \boldsymbol{v}_{h}\right) & \lesssim\|\boldsymbol{u}\|\left\|_{\Omega}\right\| \boldsymbol{v}_{h} \|_{V, \mathcal{T}} \\
b_{h}\left(q_{h}, \boldsymbol{u}\right) & \lesssim\left\|q_{h}\right\|_{Q, \mathcal{T}}\|\boldsymbol{u}\|_{\Omega}
\end{aligned}
$$

and

$$
b_{h}\left(p, \boldsymbol{v}_{h}\right) \lesssim\left(\left\|\mu^{-\frac{1}{2}} p\right\|_{0, \Omega}^{2}+\left\|\mu^{-\frac{1}{2}} p\right\|_{-1 / 2, h, \Gamma}^{2}\right)^{1 / 2}\left\|\boldsymbol{v}_{h}\right\|_{V, \mathcal{T}}
$$

We conclude by once again applying the Cauchy-Schwarz inequality.

By the symmetry of $S_{h}[(\cdot, \cdot),(\cdot, \cdot)]$, the second claim is an immediate consequence of the Cauchy-Schwarz inequality.

Lemma 4.2 (Coercivity of $a_{h}(\cdot, \cdot)$ and inf-sup stability of $\left.b_{h}(\cdot, \cdot)\right)$. There exists $c_{g}>0$ so that for any $\boldsymbol{v}_{h} \in V_{h}$ and $q_{h} \in Q_{h}$ there holds,

$$
c_{g}\left\|\boldsymbol{v}_{h}\right\|_{V, \mathcal{T}}^{2} \leq a_{h}\left(\boldsymbol{v}_{h}, \boldsymbol{v}_{h}\right)+g_{h}\left[\left(\boldsymbol{v}_{h}, 0\right),\left(\boldsymbol{v}_{h}, 0\right)\right],
$$

and with $\boldsymbol{v}_{p h}$ as in $(3.5)-(3.6)$

$$
c_{g} c_{p 2}\left\|q_{h}\right\|_{Q, \mathcal{T}}^{2} \leq b_{h}\left(q_{h}, \boldsymbol{v}_{p h}\right)+s\left(q_{h}, q_{h}\right)+g_{h}\left[\left(0, q_{h}\right),\left(0, q_{h}\right)\right] .
$$

Proof. First note that

$$
a_{h}\left(\boldsymbol{v}_{h}, \boldsymbol{v}_{h}\right)=\left\|\mu^{\frac{1}{2}} \nabla \boldsymbol{v}_{h}\right\|_{\Omega}^{2}-2\left\|\mu^{\frac{1}{2}} \boldsymbol{v}_{h}\right\|_{1 / 2, h, \Gamma}\left\|\mu^{\frac{1}{2}} \nabla \boldsymbol{v}_{h} \boldsymbol{n}\right\|_{-1 / 2, h, \Gamma}+\gamma_{\mu}\left\|\mu^{\frac{1}{2}} \boldsymbol{v}_{h}\right\|_{1 / 2, h, \Gamma}^{2},
$$

where $\gamma_{\mu}$ is a free parameter. Under assumptions A1-A3 the following inequality holds with $C_{T}$ independent of the intersection of $\partial \Omega$ with the mesh,

$$
\left\|\boldsymbol{\nabla} \boldsymbol{v}_{h} \boldsymbol{n}\right\|_{-1 / 2, h, \Gamma} \leq C_{T}\left\|\boldsymbol{\nabla} \boldsymbol{v}_{h}\right\|_{0, \Omega_{\mathcal{T}}}
$$

The reason this trace inequality holds independently of the interface position is that the norm in the right hand side is taken over all of $\Omega_{\mathcal{T}}$. Now recall the property (3.4) of the ghost penalty term. Using the above trace inequality followed by the arithmetic-geometric inequality we arrive at the relation

$$
a_{h}\left(\boldsymbol{v}_{h}, \boldsymbol{v}_{h}\right)+g\left[\left(\boldsymbol{v}_{h}, 0\right),\left(\boldsymbol{v}_{h}, 0\right)\right] \geq c_{g}\left\|\mu^{\frac{1}{2}} \nabla \boldsymbol{v}_{h}\right\|_{0, \Omega_{\mathcal{T}}}^{2}-\frac{1}{2} c_{g}\left\|\mu^{\frac{1}{2}} \nabla \boldsymbol{v}_{h}\right\|_{0, \Omega_{\mathcal{T}}}^{2}+\left(\gamma_{\mu}-2 C_{T}^{2} c_{g}^{-1}\right)\left\|\mu^{\frac{1}{2}} \boldsymbol{v}_{h}\right\|_{1 / 2, h, \Gamma}^{2} .
$$

Choosing $\gamma_{\mu}>2 C_{T}^{2} c_{g}^{-1}$ proves the claim. The second inequality, (4.5) is immediate by combining (3.4), (3.5) and (3.6).

We will now prove a global version of the inf-sup condition directly on the form $B_{h}[(\cdot, \cdot),(\cdot, \cdot)]$. The proof of the inf-sup condition works on a truncated domain, without the boundary. Once inf-sup control has been established in the interior of the domain stability up to the mesh boundary is obtained using the ghost penalty term. This means that within the domain $\Omega \backslash G_{h}$ we must have surjectivity of the divergence operator. In order for this to hold, the domain boundaries must be Lipschitz continuous uniformly in $h$. For any given mesh $\mathcal{T}_{h}$, the boundary $\Omega \backslash G_{h}$ is polygonal and hence Lipschitz. The uniformity in $h$ follows from the Lipschitz continuity of $\Gamma$ and the shape regularity of the mesh family $\left\{\mathcal{T}_{h}\right\}_{h}$.

In view of this preliminary discussion we may prove the main result.

Theorem 4.3. Let $\left(\boldsymbol{u}_{h}, p_{h}\right) \in V_{h} \times Q_{h}$. Then

$$
c_{s}\left\|\left(\boldsymbol{u}_{h}, p_{h}\right)\right\|_{h} \leq \sup _{\left(\boldsymbol{v}_{h}, q_{h}\right) \in V_{h} \times Q_{h}} \frac{B_{h}\left[\left(\boldsymbol{u}_{h}, p_{h}\right),\left(\boldsymbol{v}_{h}, q_{h}\right)\right]+S_{h}\left[\left(\boldsymbol{u}_{h}, p_{h}\right),\left(\boldsymbol{v}_{h}, q_{h}\right)\right]}{\left\|\left(\boldsymbol{v}_{h}, q_{h}\right)\right\| \|_{h}} .
$$


Proof. First by Lemma 4.2 there holds

$$
c_{g}\left\|\left|\left(\boldsymbol{u}_{h}, 0\right)\right|\right\|_{h}^{2} \leq B_{h}\left[\left(\boldsymbol{u}_{h}, p_{h}\right),\left(\boldsymbol{u}_{h}, p_{h}\right)\right]+S_{h}\left[\left(\boldsymbol{u}_{h}, p_{h}\right),\left(\boldsymbol{u}_{h}, 0\right)\right] .
$$

Consider the function $\boldsymbol{v}_{p h}$ of the stability relation (3.5) and (3.6) and observe that, again using the arithmeticgeometric inequality,

$$
\begin{aligned}
a_{h}\left(\boldsymbol{u}_{h}, \boldsymbol{v}_{p h}\right) & \leq\left\|\mu^{1 / 2} \nabla \boldsymbol{u}_{h}\right\|_{0, \Omega \backslash G_{h}}\left\|\mu^{1 / 2} \nabla \boldsymbol{v}_{p h}\right\|_{0, \Omega \backslash G_{h}} \\
& \leq\left\|\mu^{1 / 2} \nabla \boldsymbol{u}_{h}\right\|_{0, \Omega \backslash G_{h}} c_{p 1}\left\|\mu^{-1 / 2} p_{h}\right\|_{0, \Omega \backslash G_{h}} \\
& \leq c_{p 1}^{2} /\left(c_{g} c_{p 2}\right)\left\|\mu^{1 / 2} \nabla \boldsymbol{u}_{h}\right\|_{0, \Omega \backslash G_{h}}^{2}+\frac{1}{4} c_{g} c_{p 2}\left\|p_{h}\right\|_{Q, \mathcal{T}}^{2}
\end{aligned}
$$

and, using also (3.2),

$$
\begin{aligned}
S_{h}\left[\left(\boldsymbol{u}_{h}, p_{h}\right),\left(\boldsymbol{v}_{p h}, 0\right)\right] & \leq \gamma_{g} \mu g_{u}\left(\boldsymbol{u}_{h}, \boldsymbol{u}_{h}\right)^{\frac{1}{2}} g_{u}\left(\boldsymbol{v}_{p h}, \boldsymbol{v}_{p h}\right)^{\frac{1}{2}} \\
& \leq \gamma_{g} \mu^{1 / 2} g_{u}\left(\boldsymbol{u}_{h}, \boldsymbol{u}_{h}\right)^{\frac{1}{2}} c_{S}\left\|\nabla \boldsymbol{v}_{p h}\right\|_{0, \Omega_{\mathcal{T}}} \\
& =\gamma_{g} \mu^{1 / 2} g_{u}\left(\boldsymbol{u}_{h}, \boldsymbol{u}_{h}\right)^{\frac{1}{2}} c_{S}\left\|\nabla \boldsymbol{v}_{p h}\right\|_{0, \Omega \backslash G_{h}} \\
& \leq \gamma_{g} g_{u}\left(\boldsymbol{u}_{h}, \boldsymbol{u}_{h}\right)^{\frac{1}{2}} c_{S} c_{p 1} \mu^{-1 / 2}\left\|p_{h}\right\|_{0, \Omega \backslash G_{h}} \\
& \leq \gamma_{g}^{2}\left(c_{S} c_{p 1}\right)^{2} /\left(c_{g} c_{p 2}\right) g_{u}\left(\boldsymbol{u}_{h}, \boldsymbol{u}_{h}\right)+\frac{1}{4} c_{g} c_{p 2}\left\|p_{h}\right\|_{Q, \mathcal{T}}^{2}
\end{aligned}
$$

Using this inequality together with the stability conditions (3.5) and (3.6) and with (4.5) we have,

$$
\begin{aligned}
& \frac{1}{2} c_{g} c_{p 2}\left\|p_{h}\right\|_{Q, \mathcal{T}}^{2}-c_{p 1}^{2} /\left(c_{g} c_{p 2}\right)\left\|\mu^{1 / 2} \nabla \boldsymbol{u}_{h}\right\|_{0, \Omega \backslash G_{h}}^{2}-\left(\gamma_{g} c_{S} c_{p 1}\right)^{2} /\left(c_{g} c_{p 2}\right) g_{u}\left(\boldsymbol{u}_{h}, \boldsymbol{u}_{h}\right)-s_{h}\left(p_{h}, p_{h}\right) \\
& \leq B_{h}\left[\left(\boldsymbol{u}_{h}, p_{h}\right),\left(\boldsymbol{v}_{p h}, p_{h}\right)\right]+S_{h}\left[\left(\boldsymbol{u}_{h}, p_{h}\right),\left(\boldsymbol{v}_{p h}, 0\right)\right] .
\end{aligned}
$$

It follows that by taking $\boldsymbol{v}_{h}=\boldsymbol{u}_{h}+\eta \boldsymbol{v}_{p h}, q_{h}=p_{h}$ with $\eta<c_{g} c_{p 2} /\left(2 c_{p 1}^{2}\right) \max \left(1,\left(\gamma_{g} c_{S}\right)^{-2}\right)$ we have with $c_{\eta}>0$

$$
c_{\eta}\left\|\left(\boldsymbol{u}_{h}, p_{h}\right)\right\|_{h}^{2} \leq B_{h}\left[\left(\boldsymbol{u}_{h}, p_{h}\right),\left(\boldsymbol{u}_{h}+\eta \boldsymbol{v}_{p h}, p_{h}\right)\right]+S_{h}\left[\left(\boldsymbol{u}_{h}, p_{h}\right),\left(\boldsymbol{u}_{h}+\eta \boldsymbol{v}_{p h}, p_{h}\right)\right],
$$

provided $\gamma_{p}$ is positive and $\gamma_{g}$ is chosen big enough. To conclude, we only need to prove the stability estimate

$$
\left\|\left(\boldsymbol{u}_{h}+\eta \boldsymbol{v}_{p h}, p_{h}\right)\right\|_{h} \lesssim\|\|\left(\boldsymbol{u}_{h}, p_{h}\right) \|_{h} .
$$

This however follows immediately observing that

$$
\left\|\left(\boldsymbol{u}_{h}+\eta \boldsymbol{v}_{p h}, p_{h}\right)\right\|_{h} \leq\|\|\left(\boldsymbol{u}_{h}, p_{h}\right)\left\|_{h}+\right\| \mid\left(\eta \boldsymbol{v}_{p h}, 0\right) \|_{h}
$$

and, since $S\left[\left(\boldsymbol{v}_{p h}, 0\right),\left(\boldsymbol{v}_{p h}, 0\right)\right]=\gamma_{g} \mu g_{u}\left(\boldsymbol{v}_{p h}, \boldsymbol{v}_{p h}\right)$ and $\operatorname{supp}\left(\boldsymbol{v}_{p h}\right)=\Omega \backslash G_{h}$, we have using (3.2),

$$
\left\|\left(\eta \boldsymbol{v}_{p h}, 0\right)\right\|_{h}^{2}=\eta^{2}\left\|\mu^{\frac{1}{2}} \nabla \boldsymbol{v}_{p h}\right\|_{0, \Omega \backslash G_{h}}^{2}+\eta^{2} \gamma_{g} g_{u}\left(\boldsymbol{v}_{p h}, \boldsymbol{v}_{p h}\right) \lesssim \eta^{2}\left\|\mu^{\frac{1}{2}} \nabla \boldsymbol{v}_{p h}\right\|_{0, \Omega \backslash G_{h}}^{2} .
$$

Then, by the stability of $\boldsymbol{v}_{p h},(3.5)$, it follows that

$$
\left\|\left(\eta \boldsymbol{v}_{p h}, 0\right)\right\|_{h}^{2} \lesssim \eta^{2}\left(1+\gamma_{g}\right) c_{p 1}^{2} \mu^{-1}\left\|p_{h}\right\|_{0, \Omega \backslash G_{h}}^{2} \leq\left(c_{\eta}^{\prime}\right)^{2}\left\|\left(\boldsymbol{u}_{h}, p_{h}\right)\right\|_{h}^{2} .
$$

We conclude by combining (4.8) and (4.9), observing that $c_{s}=c_{\eta}\left(1+c_{\eta}^{\prime}\right)^{-1}$.

Remark 4.4. Note that since the inf-sup condition is proved in the norm $\|(\cdot, \cdot)\|_{h}$ that gives control of the velocity and the pressure over all of $\Omega_{\mathcal{T}}$, and we have continuity of $B_{h}[(\cdot, \cdot),(\cdot, \cdot)]$ on discrete spaces in the same norm, it is possible to prove that the condition number of the matrix is bounded independent of the interface/ mesh intersection following [14]. We omit the details. 


\section{Approximation properties}

We need to show that our approximating spaces $V_{h}$ and $Q_{h}$ have optimal approximation properties on suitable Hilbert spaces $\left[H^{\alpha}(\Omega)\right]^{2}$ and $H^{\beta}(\Omega)$, respectively, in the norms $\|(\cdot, \cdot)\| \|_{*}$ and $\|(\cdot, \cdot)\| \|_{h}$. This follows from some minor modifications of the analysis in [21]. We construct an interpolant of $(\boldsymbol{v}, q)$ by standard interpolants of $\left[H^{k+1}\right]^{2} \times H^{m}$-extensions of $(\boldsymbol{v}, q)$ as follows. Choose extension operators $\mathrm{E}^{s}: H^{s}(\Omega) \rightarrow H^{s}\left(\Omega_{\mathcal{T}}\right)$ such that $\left.\left(\mathrm{E}^{k+1} \boldsymbol{v}, \mathrm{E}^{m} q\right)\right|_{\Omega}=(\boldsymbol{v}, q)$ and

$$
\left\|\mathrm{E}^{s} w\right\|_{s, \Omega_{\mathcal{T}}} \lesssim\|w\|_{s, \Omega_{i}}, \quad \forall w \in H^{s}(\Omega), s=0, \ldots, \max (\alpha, \beta),
$$

cf. Dautray and Lions [18]. Let $I_{h}: H^{1}\left(\Omega_{\mathcal{T}}\right) \rightarrow V_{h}$ be a standard quasi interpolant. We will use the same interpolant for velocities and pressures, in the latter case $I_{h}^{*}: H^{1}\left(\Omega_{\mathcal{T}}\right) \rightarrow Q_{h}$. We define

$$
\boldsymbol{v}^{*}:=\mathrm{E}^{\alpha} \boldsymbol{v} \text { and } I_{h}^{*} \boldsymbol{v}:=I_{h} \boldsymbol{v}^{*}
$$

and similarly for the pressure

$$
q^{*}:=\mathrm{E}^{\beta} q \text { and } I_{h}^{*} q:=I_{h} q^{*} .
$$

Using these definitions we prove the approximation results necessary for the analysis.

Theorem 5.1. The following approximation estimates hold for the interpolation operator defined in (5.2) and (5.3)

$$
\left\|\boldsymbol{v}^{*}-I_{h}^{*} \boldsymbol{v}\right\|_{V, \Omega_{\mathcal{T}}}+\left\|q^{*}-I_{h}^{*} q\right\|_{Q, \Omega_{\mathcal{T}}} \lesssim h^{r-1} \mu^{\frac{1}{2}}\|\boldsymbol{v}\|_{r, \Omega}+h^{s} \mu^{-\frac{1}{2}}\|p\|_{s, \Omega},
$$

and

$$
\left\|\mid\left(\boldsymbol{v}-I_{h}^{*} \boldsymbol{v}, q-I_{h}^{*} q\right)\right\|_{*} \lesssim h^{r-1} \mu^{\frac{1}{2}}\|\boldsymbol{v}\|_{r, \Omega}+h^{s} \mu^{-\frac{1}{2}}\|p\|_{s, \Omega},
$$

with $r=\min (k+1, \alpha)$ and $s=\min (m+1, \beta)$ and for all $\boldsymbol{v} \in\left[H_{0}^{1}(\Omega) \cap H^{\alpha}(\Omega)\right]^{2}$ and $p \in L_{0}^{2}(\Omega) \cap H^{\beta}(\Omega)$.

For the proof of this we need the following variant of a trace inequality on a reference element that we recall from [21] and state here without proof.

Lemma 5.2. Map a triangle $K \in G_{h}$ onto the unit reference triangle $\tilde{K}$ by an affine map and denote by $\tilde{\Gamma}_{\tilde{K}}$ the corresponding image of $\Gamma_{K}$. Under the assumptions A1-A3 of Section 3 there exists a constant $C$, depending on $\Gamma$ but independent of the mesh, such that

$$
\|w\|_{0, \tilde{\Gamma}_{\tilde{K}}}^{2} \leq C\|w\|_{0, \tilde{K}}\|w\|_{1, \tilde{K}}, \quad \forall w \in H^{1}(\tilde{K}) .
$$

Proof. (Thm. 5.1) Since the mesh is non-degenerate, it follows from Lemma 5.2, scaled by the map from the reference triangle, that for $s \in \mathbb{R}$

$$
h_{K}^{-s}\|w\|_{0, \Gamma_{K}}^{2} \leq C\left(h_{K}^{-1-s}\|w\|_{0, K}^{2}+h_{K}^{1-s}\|w\|_{1, K}^{2}\right), \quad \forall w \in H^{1}(K) .
$$

Hence, using once standard interpolation estimates, there holds

$$
\begin{aligned}
h_{K}^{-1}\left\|\boldsymbol{v}-I_{h}^{*} \boldsymbol{v}\right\|_{0, \Gamma_{K}}^{2} & \leq C\left(h_{K}^{-2}\left\|\boldsymbol{v}^{*}-I_{h} \boldsymbol{v}^{*}\right\|_{0, K}^{2}+\left\|\boldsymbol{v}^{*}-I_{h} \boldsymbol{v}^{*}\right\|_{1, K}^{2}\right) \\
& \leq C h_{K}^{r-1}\left\|\boldsymbol{v}^{*}\right\|_{r, K}^{2} .
\end{aligned}
$$

As a consequence, by the stability of the extension operator,

$$
\mu^{\frac{1}{2}}\left\|\boldsymbol{v}-I_{h}^{*} \boldsymbol{v}\right\|_{1 / 2, h, \Gamma} \lesssim \mu^{\frac{1}{2}} h_{K}^{r-1}\left\|\boldsymbol{v}^{*}\right\|_{r, \Omega_{\mathcal{T}}}^{2} \lesssim \mu^{\frac{1}{2}} h_{K}^{r-1}\|\boldsymbol{v}\|_{r, \Omega}^{2} .
$$


The first claim (5.4) then follows by standard interpolation estimates for the operator $I_{h}^{*}$, and the stability of the extension operator. Since

$$
\begin{aligned}
\left\|\left(\boldsymbol{v}-I_{h}^{*} \boldsymbol{v}, q-I_{h}^{*} q\right)\right\|_{*} \lesssim & \left\|\boldsymbol{v}^{*}-I_{h}^{*} \boldsymbol{v}\right\|_{V, \Omega_{\mathcal{T}}}+\left\|q^{*}-I_{h}^{*} q\right\|_{Q, \Omega_{\mathcal{T}}} \\
& +\left\|\mu^{\frac{1}{2}} \boldsymbol{\nabla}\left(\boldsymbol{v}-I_{h}^{*} \boldsymbol{v}\right) \boldsymbol{n}\right\|_{-1 / 2, h, \Gamma}+\left\|\mu^{-\frac{1}{2}}\left(q-I_{h}^{*} q\right)\right\|_{-1 / 2, h, \Gamma}
\end{aligned}
$$

we only need to upper bound the two remaining contributions in the right hand side. They may both be treated similarly as $\left\|\boldsymbol{v}-I_{h}^{*} \boldsymbol{v}\right\|_{1 / 2, h, \Gamma}$ and we give the details only for the viscous fluxes. Lemma 5.2 applied to $\boldsymbol{\nabla} \boldsymbol{w} \boldsymbol{n}$ and scaling gives

$$
h_{K}\|\boldsymbol{\nabla} \boldsymbol{w} \boldsymbol{n}\|_{0, \Gamma_{K}}^{2} \leq C\left(\|\boldsymbol{w}\|_{1, K}^{2}+h_{K}^{2}\|\boldsymbol{w}\|_{2, K}^{2}\right), \quad \forall \boldsymbol{w} \in\left[H^{2}(K)\right]^{2} .
$$

Using this result applied to $\boldsymbol{w}=\boldsymbol{v}^{*}-I_{h} \boldsymbol{v}^{*}$ and again standard interpolation estimates, it follows that

$$
\begin{aligned}
h_{K}\left\|\boldsymbol{\nabla}\left(\boldsymbol{v}-I_{h}^{*} \boldsymbol{v}\right) \boldsymbol{n}\right\|_{0, \Gamma_{K}}^{2} & \lesssim h_{K}\left\|\boldsymbol{\nabla}\left(\boldsymbol{v}-I_{h}^{*} \boldsymbol{v}\right)\right\|_{0, \Gamma_{K}}^{2} \\
& \lesssim\left(h_{K}\left\|\boldsymbol{v}^{*}-I_{h} \boldsymbol{v}^{*}\right\|_{1, K}^{2}+h_{K}^{2}\left\|\boldsymbol{v}^{*}-I_{h} \boldsymbol{v}^{*}\right\|_{2, K}^{2}\right) \\
& \lesssim h_{K}^{2(r-1)}\left\|\boldsymbol{v}^{*}\right\|_{r, K}^{2} .
\end{aligned}
$$

Summing again the contributions from $K \in G_{h}$, we deduce from (5.1) that

$$
\left\|\boldsymbol{\nabla}\left(\boldsymbol{v}-I_{h}^{*} \boldsymbol{v}\right) \boldsymbol{n}\right\|_{-1 / 2, h, \Gamma} \lesssim h^{r-1}\|\boldsymbol{v}\|_{r, \Omega} .
$$

Similarly for the traces of the pressures

$$
\left\|\left(q-I_{h}^{*} q\right)\right\|_{-1 / 2, h, \Gamma} \lesssim h^{s}\|q\|_{s, \Omega} .
$$

To prove optimal convergence the stabilization operator $S_{h}[(\cdot, \cdot),(\cdot, \cdot)]$ must be weakly consistent to the right order. More precisely we will assume that the following Lemma holds.

Lemma 5.3. For all $q \in H^{\beta}(\Omega), \boldsymbol{v} \in\left[H^{\alpha}(\Omega)\right]^{2}$ there holds

$$
S_{h}\left[\left(I_{h}^{*} \boldsymbol{v}, I_{h}^{*} q\right),\left(I_{h}^{*} \boldsymbol{v}, I_{h}^{*} q\right)\right] \lesssim h^{2(r-1)} \mu\|\boldsymbol{v}\|_{r, \Omega}^{2}+\mu^{-1} h^{2 s}\|q\|_{s, \Omega}^{2},
$$

with $r=\min (k+1, \alpha)$ and $s=\min (m+1, \beta)$.

For a proof of this result we refer to $[5,8,12,13,15,16]$ for the pressure stabilization part and to $[14,16]$ for the ghost penalty part.

\section{A PRIORI ERROR ESTIMATES}

Using the stability and continuity properties derived in the previous sections it is now straightforward to show optimal convergence orders of the error.

Theorem 6.1. Assume that the solution $(\boldsymbol{u}, p)$ to problem $(2.2)$ resides in $\left[H^{\alpha}(\Omega)\right]^{2} \times H^{\beta}(\Omega)$. Then the finite element solution (3.1) satisfies the error estimate

$$
\left\|\boldsymbol{u}-\boldsymbol{u}_{h}\right\|_{V}+\left\|p-p_{h}\right\|_{Q} \lesssim h^{r-1} \mu^{\frac{1}{2}}\|\boldsymbol{u}\|_{r, \Omega}+h^{s} \mu^{-\frac{1}{2}}\|p\|_{s, \Omega},
$$

with $r=\min (k+1, \alpha)$ and $s=\min (m+1, \beta)$. 
Proof. First we set $\boldsymbol{\eta}_{h}=\boldsymbol{u}_{h}-I_{h}^{*} \boldsymbol{u}$ and $\zeta_{h}=p_{h}-I_{h}^{*} p$ and note that

$$
\begin{aligned}
\left\|\boldsymbol{u}-\boldsymbol{u}_{h}\right\|_{V}+\left\|p-p_{h}\right\|_{Q} & \leq\left\|\mathrm{E}^{\alpha} \boldsymbol{u}-\boldsymbol{u}_{h}\right\|_{V, \Omega_{\mathcal{T}}}+\left\|\mathrm{E}^{\beta} p-p_{h}\right\|_{Q, \Omega_{\mathcal{T}}} \\
& \leq\left\|\mathrm{E}^{\alpha} \boldsymbol{u}-I_{h}^{*} \boldsymbol{u}\right\|_{V, \Omega_{\mathcal{T}}}+\left\|\mathrm{E}^{\beta} p-I_{h}^{*} p\right\|_{Q, \Omega_{\mathcal{T}}}+\left\|\left(\boldsymbol{\eta}_{h}, \zeta_{h}\right)\right\|_{h} .
\end{aligned}
$$

In view of Theorem 5.1 we only need to show the inequality for $\left\|\left(\boldsymbol{\eta}_{h}, \zeta_{h}\right)\right\|_{h}$. By Theorem 4.3 we obtain

$$
\left\|\left(\boldsymbol{\eta}_{h}, \zeta_{h}\right)\right\|_{h} \leq \frac{1}{c_{s}} \sup \frac{B_{h}\left[\left(\boldsymbol{\eta}_{h}, \zeta_{h}\right),\left(\boldsymbol{v}_{h}, q_{h}\right)\right]+S_{h}\left[\left(\boldsymbol{\eta}_{h}, \zeta_{h}\right),\left(\boldsymbol{v}_{h}, q_{h}\right)\right]}{\left\|\left(\boldsymbol{v}_{h}, q_{h}\right)\right\|_{h}},
$$

and by Galerkin orthogonality

$$
\left\|\left(\boldsymbol{\eta}_{h}, \zeta_{h}\right)\right\|_{h} \leq \frac{1}{c_{s}} \sup \frac{B_{h}\left[\left(\boldsymbol{u}-I_{h}^{*} \boldsymbol{u}, p-I_{h}^{*} p\right),\left(\boldsymbol{v}_{h}, q_{h}\right)\right]-S_{h}\left[\left(I_{h}^{*} \boldsymbol{u}, I_{h}^{*} p\right),\left(\boldsymbol{v}_{h}, q_{h}\right)\right]}{\left\|\left(\boldsymbol{v}_{h}, q_{h}\right)\right\|_{h}} .
$$

The suprema above are taken over $\left(\boldsymbol{v}_{h}, q_{h}\right) \in V_{h} \times Q_{h}$.

Applying now the continuity of $B_{h}[(\cdot, \cdot),(\cdot, \cdot)],(4.2)$, and $S_{h}[(\cdot, \cdot),(\cdot, \cdot)](4.3)$, we obtain the best approximation type inequality

$$
\left\|\left(\boldsymbol{\eta}_{h}, \zeta_{h}\right)\right\|_{h} \lesssim\left\|\mid\left(\boldsymbol{u}-I_{h}^{*} \boldsymbol{u}, p-I_{h}^{*} p\right)\right\|_{*}+S_{h}\left[\left(I_{h}^{*} \boldsymbol{u}, I_{h}^{*} p\right),\left(I_{h}^{*} \boldsymbol{u}, I_{h}^{*} p\right)\right]^{\frac{1}{2}}
$$

We conclude using the approximation results of Theorem 5.1 and (5.9)

Corollary 6.2. Under the same assumptions as in Theorem 6.1 there holds

$$
S_{h}\left[\left(\boldsymbol{u}_{h}, p_{h}\right),\left(\boldsymbol{u}_{h}, p_{h}\right)\right]^{\frac{1}{2}} \lesssim h^{r-1} \mu^{\frac{1}{2}}\|\boldsymbol{u}\|_{r, \Omega}+h^{s} \mu^{-\frac{1}{2}}\|p\|_{u, \Omega}
$$

and

$$
\left\|\left(\boldsymbol{u}-\boldsymbol{u}_{h}, p-p_{h}\right)\right\|_{*} \lesssim h^{r-1} \mu^{\frac{1}{2}}\|\boldsymbol{u}\|_{r, \Omega}+h^{u} \mu^{-\frac{1}{2}}\|p\|_{u, \Omega},
$$

with $r=\min (k+1, \alpha)$ and $s=\min (m+1, \beta)$.

Proof. The proof is immediate since by the triangle inequality

$$
\begin{aligned}
S_{h}\left[\left(\boldsymbol{u}_{h}, p_{h}\right),\left(\boldsymbol{u}_{h}, p_{h}\right)\right]^{\frac{1}{2}} \leq & S_{h}\left[\left(\boldsymbol{u}_{h}-I_{h}^{*} \boldsymbol{u}, p_{h}-I_{h}^{*} p\right),\left(\boldsymbol{u}_{h}-I_{h}^{*} \boldsymbol{u}, p_{h}-I_{h}^{*} p\right)\right]^{\frac{1}{2}} \\
& +S_{h}\left[\left(I_{h}^{*} \boldsymbol{u}, I_{h}^{*} p\right),\left(I_{h}^{*} \boldsymbol{u}, I_{h}^{*} p\right)\right]^{\frac{1}{2}}
\end{aligned}
$$

and

$$
\begin{aligned}
\left\|\left(\boldsymbol{u}-\boldsymbol{u}_{h}, p-p_{h}\right)\right\|_{*} & \leq\left\|\left(\boldsymbol{u}-I_{h}^{*} \boldsymbol{u}, p-I_{h}^{*} p\right)\right\|\left\|_{*}+\right\|\left(\boldsymbol{u}_{h}-I_{h}^{*} \boldsymbol{u}, p_{h}-I_{h}^{*} p\right) \|_{*} \\
& \lesssim\|\|\left(\boldsymbol{u}-I_{h}^{*} \boldsymbol{u}, p-I_{h}^{*} p\right)\|\|_{*}+\left\|\left(\boldsymbol{u}_{h}-I_{h}^{*} \boldsymbol{u}, p_{h}-I_{h}^{*} p\right)\right\|_{h} .
\end{aligned}
$$

The conclusion follows by Theorem 6.1 and the approximation results of Theorem 5.1.

Using the Aubin-Nitsche duality argument we prove the following $L_{2}(\Omega)$-estimate for the displacements. Consider the dual adjoint problem Let $\boldsymbol{w}$ and $r$ be the solution of the problem

$$
\begin{aligned}
-\mu \Delta \boldsymbol{w}-\boldsymbol{\nabla} r & =\boldsymbol{\psi} & & \text { in } \Omega \\
\nabla \cdot \boldsymbol{w} & =0 & & \text { in } \Omega \\
\boldsymbol{w} & =0 & & \text { on } \partial \Omega .
\end{aligned}
$$

We assume that the solution of the adjoint problem enjoys the regularity

$$
\left\|\mu^{\frac{1}{2}} \boldsymbol{w}\right\|_{2, \Omega}+\left\|\mu^{-\frac{1}{2}} r\right\|_{1, \Omega} \leq c_{\mu}\|\boldsymbol{\psi}\|_{0, \Omega} .
$$


Proposition 6.3. Assume that $\alpha \geq k+1$ and $\beta \geq m \geq k-1$. Under the same assumptions as Theorem 6.1 and assuming that (6.1) and (6.2) holds. Then

$$
\left\|\boldsymbol{u}-\boldsymbol{u}_{h}\right\|_{0, \Omega} \lesssim h^{k+1}\left(\mu^{\frac{1}{2}}\|\boldsymbol{u}\|_{k+1, \Omega}+\mu^{-\frac{1}{2}}\|p\|_{k, \Omega}\right)
$$

where the hidden constant is independent of $h$, and the interface/mesh intersection, but not of the polynomial order nor of the viscosity $\mu$.

Proof. Choosing $\boldsymbol{\psi}=\boldsymbol{u}-\boldsymbol{u}_{h}$, we may write

$$
\left\|\boldsymbol{u}-\boldsymbol{u}_{h}\right\|_{0, \Omega}^{2}=a_{h}\left(\boldsymbol{u}-\boldsymbol{u}_{h}, \boldsymbol{w}\right)-b_{h}\left(\boldsymbol{u}-\boldsymbol{u}_{h}, r\right)
$$

and proceed using Galerkin orthogonality and the Cauchy-Schwartz inequality to obtain

$$
\begin{aligned}
\left\|\boldsymbol{u}-\boldsymbol{u}_{h}\right\|_{0, \Omega}^{2}= & a_{h}\left(\boldsymbol{u}-\boldsymbol{u}_{h}, \boldsymbol{w}-I_{h}^{*} \boldsymbol{w}\right)-b_{h}\left(\boldsymbol{u}-\boldsymbol{u}_{h}, r-I_{h}^{*} r\right) \\
& +b_{h}\left(I_{h}^{*} \boldsymbol{w}, p-p_{h}\right)-S_{h}\left[\left(\boldsymbol{u}_{h}, p_{h}\right),\left(I_{h}^{*} \boldsymbol{w}, I_{h}^{*} r\right)\right] \\
= & B_{h}\left[\left(\boldsymbol{u}-\boldsymbol{u}_{h}, p-p_{h}\right),\left(\boldsymbol{w}-I_{h}^{*} \boldsymbol{w}, r-I_{h}^{*} r\right)\right]-S_{h}\left[\left(\boldsymbol{u}_{h}, p_{h}\right),\left(I_{h}^{*} \boldsymbol{w}, I_{h}^{*} r\right)\right] \\
\leq & \left\|\left(\boldsymbol{u}-\boldsymbol{u}_{h}, p-p_{h}\right)\right\|\left\|_{*}\right\|\left(\boldsymbol{w}-I_{h}^{*} \boldsymbol{w}, \boldsymbol{w}, r-I_{h}^{*} r\right)\|\|_{h} . \\
& +S_{h}\left[\left(\boldsymbol{u}_{h}, p_{h}\right),\left(\boldsymbol{u}_{h}, p_{h}\right)\right]^{\frac{1}{2}} S_{h}\left[\left(I_{h}^{*} \boldsymbol{w}, I_{h}^{*} r\right),\left(I_{h}^{*} \boldsymbol{w}, I_{h}^{*} r\right)\right]^{\frac{1}{2}} \\
\leq & c\left(\left\|\left(\boldsymbol{u}-\boldsymbol{u}_{h}, p-p_{h}\right)\right\|_{*}+S_{h}\left[\left(\boldsymbol{u}_{h}, p_{h}\right),\left(\boldsymbol{u}_{h}, p_{h}\right)\right]^{\frac{1}{2}}\right) h\left(\mu^{\frac{1}{2}}\|\boldsymbol{w}\|_{2, \Omega}+\mu^{-\frac{1}{2}}\|r\|_{1, \Omega}\right) .
\end{aligned}
$$

The claim now follows as a consequence of Proposition 6.1, Theorem 5.1 and the regularity hypothesis (6.2).

\section{Numerical EXAMPLES}

\subsection{Convergence and stability}

We consider an example with exact solution

$$
u_{x}=20 x y^{3}, \quad u_{y}=5 x^{4}-5 y^{4}, \quad p=60 x^{2} y-20 y^{3} .
$$

Our computational domain is a disc with center at the origin. The exact velocities are used as Dirichlet data on the edge of the domain. We set $\mu=1$ and $\boldsymbol{f}=0$. The exact pressure integrates to zero, so we impose zero average of the discrete pressure. The boundary of the domain is given by the isoline of a level set function defined on a covering mesh using a piecewise linear interpolant, $c f$. Figure 1. Note that symmetry is avoided by not centering the mesh.

We compare two methods: one stabilized using piecewise linear approximations for velocity and pressure (P1P1), with the pressure stabilized in the whole domain using gradient jump; the velocity stabilization in the cut element zone is stabilized in the same fashion, as discussed in Section 3.1. The second approach is to use the MINI element of Arnold et al. [4]. This element is inf-sup stable, and so only needs stabilization in the cut element zone. For the MINI element, we choose to use the local projection method of Becker et al. [6] with projections onto linear functions on patches composed of the cut element and its cut neighbors (one projection for each cut neighbor), as suggested in Section 3.1. The Nitsche parameter was chosen fixed as $\gamma_{\mu}=10$.

In Figues 2 and 3 we show the elevation of the discrete solution for the different elements. The convergence for the different elements are shown in Figures 4, using $\gamma_{g}=\gamma_{p}=0.1$, and 5, using $\gamma_{g}=0.1$. Finally, in Figure 6 we show the effect of lowering the size of the stabilization terms (equally much). For the P1P1, the same stability term $\gamma_{p}=\gamma_{g}$ is used in the whole of the domain, so this leads to global instability, whereas for the MINI element, for which $\gamma_{p}=0$, the stability loss is confined to the cut element zone. 


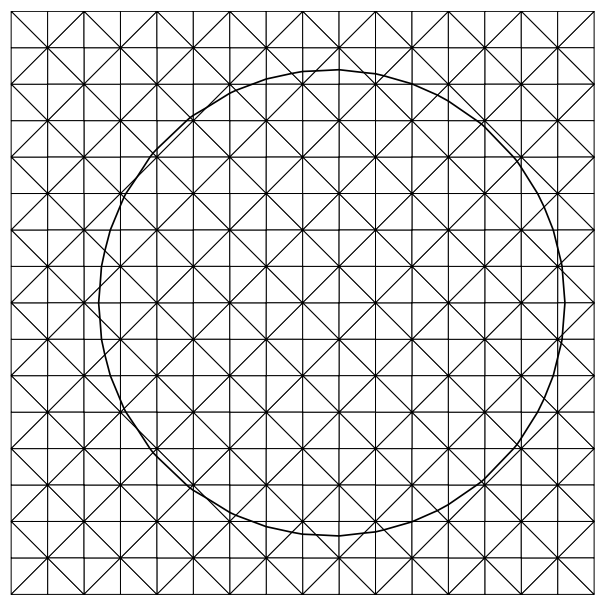

Figure 1. Level set isoline used to define the domain boundary.
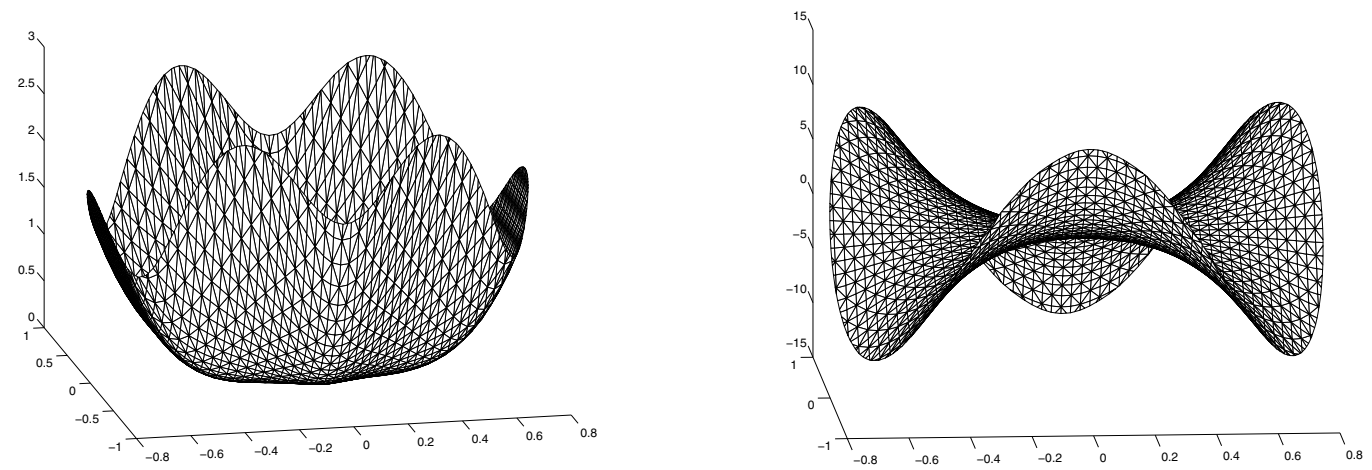

FiguRE 2. Elevation of the length of the velocity (left) and the pressure (right) for the stabilized $P 1-P 1$ method.
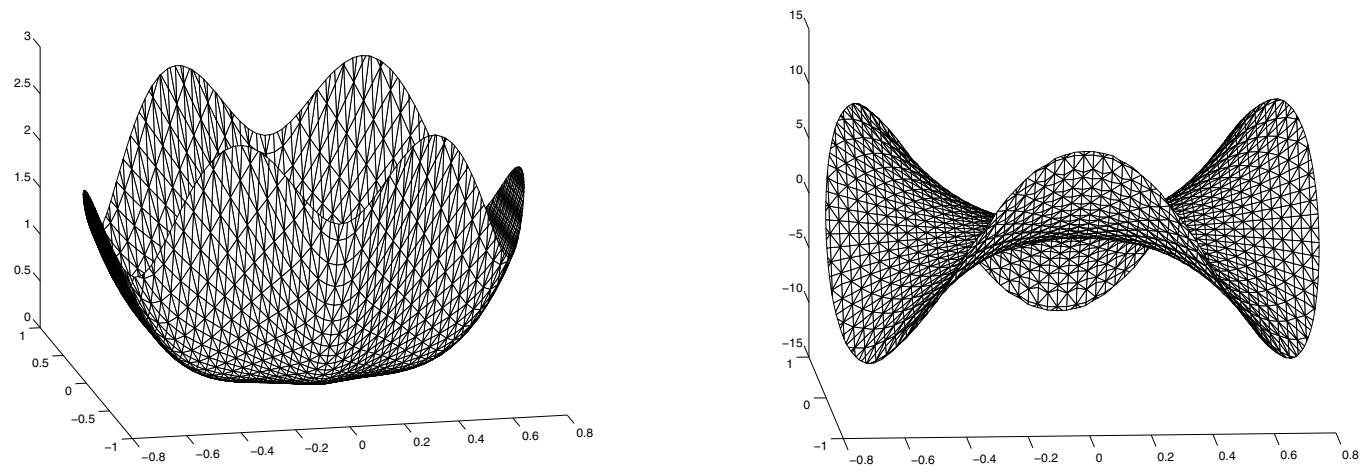

FIGURE 3. Elevation of the length of the velocity (left) and the pressure (right) for the MINI element. 


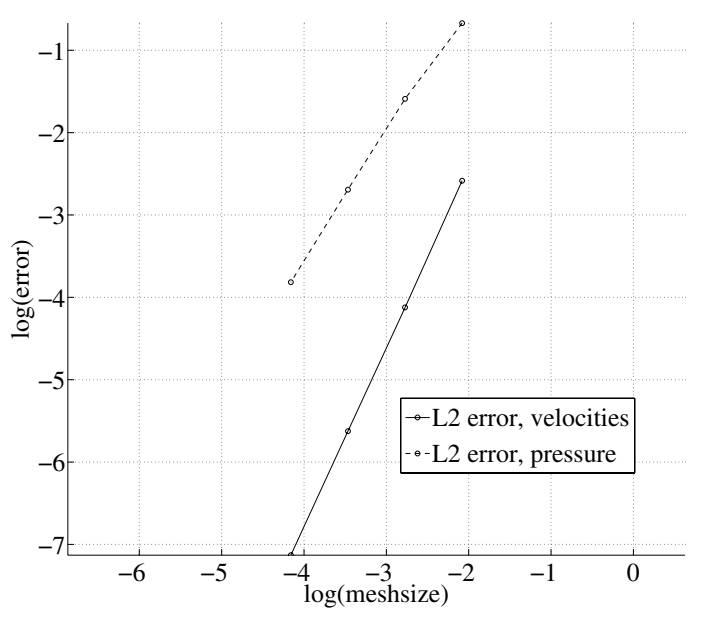

Figure 4. Convergence using the P1P1 element.

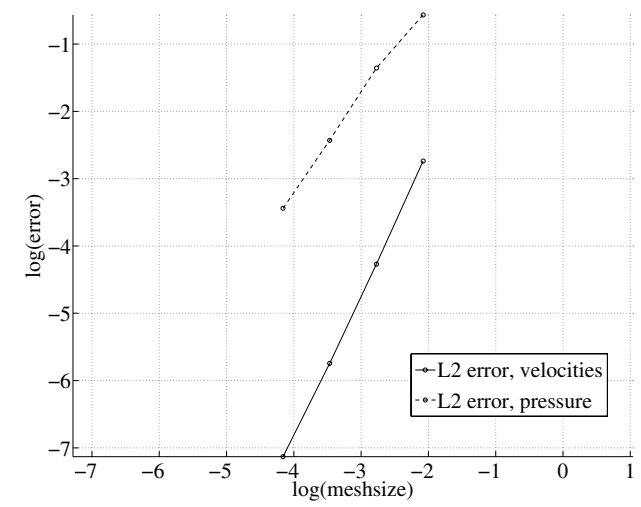

FiguRE 5. Convergence using the MINI element.
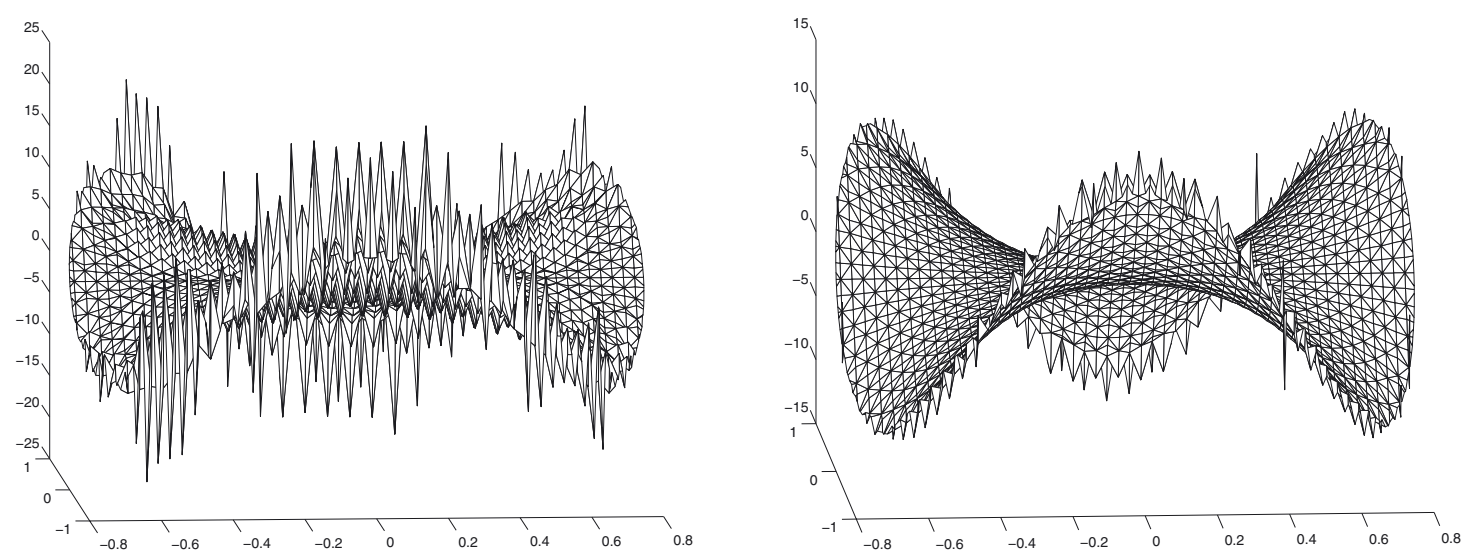

Figure 6. Elevation of the pressure for the P1P1 (left) and MINI (right) in the case of insufficient stabilization. 


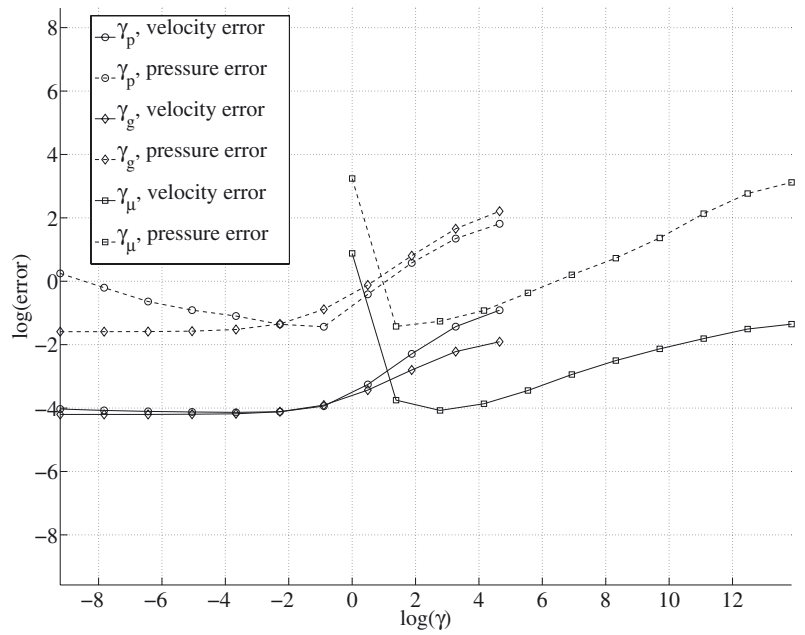

FiguRE 7 . Sensitivity of $L_{2}$ errors with respect to the stabilization parameters.

\subsection{Sensitivity with respect to the stabilization parameters}

We also give a graph illustrating the sensitivity concerning convergence with respect to the parameters $\gamma_{\mu}$, $\gamma_{p}$, and $\gamma_{g}$. In Figure 7 we show the robustness of the velocity and pressure errors in $L_{2}(\Omega)$ with respect to the size of the different parameters. The variation of the parameters is carried out around a base state of $\gamma_{\mu}=10$, $\gamma_{p}=0.1$, and $\gamma_{g}=0.1$. We are working with the stabilized element, so as $\gamma_{p} \rightarrow 0$ we see an increase of the error, whereas the function of $\gamma_{g}$ is subsumed by $\gamma_{p}$ in the case $\gamma_{g} \rightarrow 0$. Note in particular the low sensitivity of the errors with respect to several orders of magnitude of the stabilization parameters.

\section{REFERENCES}

[1] S. Amdouni, K. Mansouri, Y. Renard, M. Arfaoui and M. Moakher, Numerical convergence and stability of mixed formulation with X-FEM cut-off. Eur. J. Comput. Mech. 21 (2012) 160-73.

[2] S. Amdouni, M. Moakher and Y. Renard, A local projection stabilization of fictitious domain method for elliptic boundary value problems. Preprint, hal.archives-ouvertes.fr: hal-00713115 (2012)

[3] Ph. Angot, A fictitious domain model for the Stokes/Brinkman problem with jump embedded boundary conditions. C.R. Math. Acad. Sci. Paris 348 (2010) 697-702.

[4] D.N. Arnold, F. Brezzi and M. Fortin, A stable finite element for the Stokes equation. Calcolo 21 (1984) $337-344$.

[5] R. Becker and M. Braack, A finite element pressure gradient stabilization for the Stokes equations based on local projections. Calcolo 38 (2001) 173-199.

[6] R. Becker, E. Burman and P. Hansbo, A finite element time relaxation method. C.R. Math. Acad. Sci. Paris 349 (2011) $353-356$.

[7] R. Becker, E. Burman and P. Hansbo, A Nitsche extended finite element method for incompressible elasticity with discontinuous modulus of elasticity. Comput. Methods Appl. Mech. Engrg. 198 (2009) 3352-3360.

[8] R. Becker and P. Hansbo, A simple pressure stabilization method for the Stokes equation. Commun. Numer. Methods Eng. 24 (2008) 1421-1430.

[9] M. Bercovier and O. Pironneau, Error estimates for finite element method solution of the Stokes problem in the primitive variables. Numer. Math. 33 (1979) 211-224.

[10] S. Bertoluzza, M. Ismail and B. Maury, Analysis of the fully discrete fat boundary method. Numer. Math. 118 (2011) 49-77.

[11] D. Boffi, F. Brezzi, L. Demkowicz, R. Durán, R. Falk and M. Fortin, Mixed finite elements, compatibility conditions, and applications. Lectures given at the C.I.M.E. Summer School held in Cetraro 2006, edited by Boffi and Lucia Gastaldi. In vol. 1939 Lect. Notes Math. Springer-Verlag, Berlin (2008).

[12] F. Brezzi and J. Pitkäranta, On the stabilization of finite element approximations of the Stokes equations, in Efficient solutions of elliptic systems (Kiel, 1984), vol. 10 of Notes Numer. Fluid Mech. Vieweg, Braunschweig (1984) 11-19.

[13] F. Brezzi and R. Falk, Stability of higher-order Hood-Taylor methods. SIAM J. Numer. Anal. 28 (1991) 581-590. 
[14] E. Burman and P. Hansbo, Fictitious domain finite element methods using cut elements: II. A stabilized Nitsche method. Appl. Numer. Math. 62 (2012) 328-341.

[15] E. Burman and P. Hansbo, Edge stabilization for the generalized Stokes problem: a continuous interior penalty method. Comput. Methods Appl. Mech. Engrg. 195 (2006) 2393-2410.

[16] E. Burman, Pressure projection stabilizations for Galerkin approximations of Stokes' and Darcy's problem. Numer. Methods Part. Differ. Eqs. 24 (2008) 127-143.

[17] E. Burman, Ghost penalty. C.R. Math. Acad. Sci. Paris 348 (2010) 1217-1220.

[18] R. Dautray and J.-L. Lions, Mathematical Analysis and Numerical Methods for Science and Technology, vol. 2. Functional and Variational Methods. Springer-Verlag, Berlin (1988)

[19] C. Dohrmann and P. Bochev, A stabilized finite element method for the Stokes problem based on polynomial pressure projections. Int. J. Numer. Methods Fluids 46 (2004) 183-201.

[20] V. Girault, R. Glowinski and T. Pan, A fictitious-domain method with distributed multiplier for the Stokes problem, in Appl. Nonlinear Anal. Kluwer/Plenum, New York (1999) 159-174.

[21] A. Hansbo and P. Hansbo, An unfitted finite element method, based on Nitsche's method, for elliptic interface problems. Comput. Methods Appl. Mech. Engrg. 47 (2009) 5537-5552.

[22] J. Haslinger and Y. Renard, A new fictitious domain approach inspired by the extended finite element method. SIAM J. Numer. Anal. 191 (2002) 1474-1499.

[23] G. Legrain, N. Moës and A. Huerta, Stability of incompressible formulations enriched with X-FEM. Comput. Methods Appl. Mech. Engrg. 197 (2008) 1835-1849.

[24] J. Nitsche, Über ein Variationsprinzip zur Lösung von Dirichlet-Problemen bei Verwendung von Teilräumen, die keinen Randbedingungen unterworfen sind. Abh. Math. Sem. Univ. Hamburg 36 (1971) 9-15. 